\title{
Development and initial validation of a new functional ability tool for juvenile dermatomyositis
}

\author{
GC Varnier ${ }^{1 *}$, A Consolaro ${ }^{1,2}$, C Malattia $^{1,2}$, AP Rao ${ }^{1}$, A Madeo $^{1}$, E Demirkaya $^{1}$, D Lazarevic ${ }^{1}$, A Civino ${ }^{3}$, A Martini ${ }^{1,2}$, \\ A Ravelli ${ }^{1,2}$
}

From 18th Pediatric Rheumatology European Society (PReS) Congress

Bruges, Belgium. 14-18 September 2011

\section{Background}

The assessment of functional ability is an essential part of the clinical evaluation of patients with juvenile dermatomyositis (JDM). However, no physical function tool specific for JDM is currently available.

\section{Objectives}

To develop and validate a new functional ability questionnaire specific for JDM, named Juvenile Myositis Functionality Scale (MyoFun).

\section{Methods}

The MyoFun assesses the ability of the child to perform 15 activities that require the use of all skeletal muscles and muscle groups. Each item is scored from 0 to 3 ( $0=$ with no difficulty; $1=$ with some difficulty; $2=$ with much difficulty; $3=$ unable to do). The total score ranges from 0 (normal physical function) to 45 (worst physical function). A parent of 27 children with JDM was asked to complete the MyoFun and the Childhood Health Assessment Questionnaire (C-HAQ) and to rate the child's overall well-being and pain intensity on a 21numbered circle visual analog scale (VAS). The attending physician assessed the child's muscle strength/function with the Kendall's Manual Muscle Testing (MMT) and the Childhood Myositis Assessment Scale (CMAS). Laboratory tests included creatine phosphokinase (CK) and lactic dehydrogenase (LDH). Construct validity of the MyoFun was examined by calculating its Spearman's correlations with the other JDM outcome measures on both cross-sectional data and change between 2

\footnotetext{
* Correspondence: giuliavarnier@yahoo.it

${ }^{1}$ Pediatria II, IRCCS G.Gaslini, Genova, Italy

Full list of author information is available at the end of the article
}

Table

\begin{tabular}{llllllll}
\hline & MMT & CMAS & C-HAQ & Well-being VAS & Pain VAS & CK & LDH \\
\hline MyoFun & -0.78 & -0.66 & 0.90 & 0.85 & 0.89 & $0.27^{*}$ & 0.43 \\
\hline
\end{tabular}

consecutive visits. Correlations were considered high, moderate or low when $>0.7,0.4-0.7$, or $<0.4$, respectively

\section{Results}

The MyoFun was found to be feasible, requiring $<5$ minutes to be completed and $<2$ minutes to be scored. The table shows the Spearman's correlations of the MyoFun score with the scores of the other JDM outcome measures.

A second visit made within 6 months from baseline visit was available for 13 patients. Spearman's correlation between the change in the MyoFun score and the change in the MMT score was -0.90 .

\section{Conclusion}

Preliminary validation analyses have shown that the MyoFun is feasible and has an excellent construct validity and is, thus, suitable for the assessment of functional ability in children with JDM.

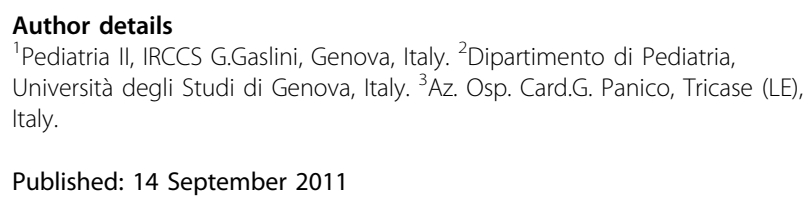

Published: 14 September 2011

\section{doi:10.1186/1546-0096-9-S1-P61}

Cite this article as: Varnier et al.: Development and initial validation of a new functional ability tool for juvenile dermatomyositis. Pediatric

Rheumatology 2011 9(Suppl 1):P61.

\section{()

(C) 2011 Varnier et al; licensee BioMed Central Ltd. This is an open access article distributed under the terms of the Creative Commons Attribution License (http://creativecommons.org/licenses/by/2.0), which permits unrestricted use, distribution, and reproduction in any medium, provided the original work is properly cited. 